\title{
Korporatismus oder Etatismus? \\ Formierung von Verhandlungspositionen zu EU-Gesetzgebungsvorhaben
}

\author{
Gerald Schneider/Daniel Finke/Konstantin Baltz
}

\section{$1 \quad$ Einleitung ${ }^{1}$}

Integrationstheoretiker sind wiederholt über die Rolle, die Interessengruppen im Prozess der Europäischen Integration spielen, gestolpert. Besonders Haas (1958, 1964) argumentiert, dass anhaltende Kooperation nur zu erreichen sei, wenn integrationistische Eliten in die Entscheidungsfindung einbezogen seien. Sandholtz/Zysman (1989) argumentieren in dieselbe Richtung. Sie zeigen, wie multinationale Firmen Regierungen zur Annahme des Binnenmarktprogramms von 1992 brachten. Diese rosigen Einschätzungen stehen im starken Kontrast zu den negativen Effekten, die Vertreter des Public-Choice-Ansatzes mit Interessengruppen assoziieren. Gillingham (1991) schreibt die Gründung der Europäischen Gemeinschaft für Kohle und Stahl den Wünschen der Kartellindustrie zu, staatliche Protektion zu erhalten. In seiner Geschichte des Integrationsprozesses bedauert er den Umschwung zu korporatistischer Entscheidungsfindung während der Amtszeit von Kommissionspräsident Delors (Gillingham 2003). Das daraus resultierende Gemisch zwischen étatistischen Interventionen und Klientelismus trug nach Ansicht des ultraliberalen Autors zu den europäischen Wachstumsproblemen bei. Das gemeinsame Problem beider Aussagen - der optimistischen und der pessimistischen Darstellung der Rolle, die Interessengruppen in der europäischen. Integration spielen - ist die oftmals mangelhafte und unsystematische empirische Grundlage.

\footnotetext{
'Eine erste Version dieses Papiers wurde auf der DVPW-Tagung „Interessenvermittlung in Politikfeldern“, MPI Köln, 17.-19. Juni 2005, vorgestellt. Dieser Buchbeitrag ist die ausführliche Fassung eines englischsprachigen Werkes derselben Autoren (Schneider/Baltz/Finke 2007). Bei der Erstellung des Manuskriptes konnten die Autoren auf die Hilfe von Lena May zählen. Für finanzielle Unterstützung möchten sie sich bei der Deutschen Forschungsgemeinschaft bedanken. Anette Arslantas, Ursula Klöpper, Christian Lau, Michael Weltin und Carl Zimanky haben den Autoren in der ersten Phase des Projektes kompetent geholfen.
} 
Da die vergleichende Literatur zur Interessenvermittlung relativ deskriptiv ist, wissen wir nicht, wie Interessengruppen und Regierungen im EUEntscheidungsprozess interagieren. Insofern ist die politikwissenschaftliche Forschung weit davon entfernt, zu verstehen, wie Interessenvermittlung in der EU funktioniert. Dieser Beitrag versucht, die Forschungslücke für einen Teilaspekt dieser Prozesse zu schließen, und zwar für die Vorverhandlungen, die im Anschluss an die Unterbreitung eines Gesetzgebungsvorschlages der EUKommission im nationalen Rahmen stattfinden. Wir werden die Formierung von Verhandlungspositionen zu 15 Vorschlägen und in vier Mitgliedsstaaten untersuchen: Finnland, Deutschland, den Niederlanden und Großbritannien. Den Annahmen in der Literatur folgend sollten für diese Staaten unterschiedliche Logiken der Interessenvermittlung vorherrschen. Während man Großbritannien mit einem pluralistischen Wettbewerb zwischen Interessengruppen assoziiert, herrscht in Kontinentaleuropa traditionell eine selektive Beteiligung einzelner privater Akteure vor. Die meisten Autoren vermuten, dass korporatistische Formen der Interessenvermittlung auch bei der EU-Politikgestaltung entstehen sollten (z.B. Grote/Schmitter 1999), wohingegen einige wenige Wissenschaftler glauben, dass konkordanzdemokratische Überlegungen innerhalb der EU zunehmend relevanter werden (Bogaards/Crepaz 2002).

Das Kapitel evaluiert die Erklärungskraft korporatistischer, konkordanzdemokratischer, pluralistischer, étatistischer und klientelistischer Interessenvermittlungsmodelle anhand ihrer Vorhersagegenauigkeit. Obwohl die komparative Literatur - angefangen bei Lehmbruch (1967) und Schmitter (1974) - den Fokus hauptsächlich auf die ersten beiden Typen richtet, gibt es Gründe, zu glauben, dass die Rolle der Regierung weit über die bloße Koordination von divergierenden Positionen hinausgeht. Wie Schneider/Baltz (2005) zeigen, besitzen Ministerien eine beachtliche diskretionäre Macht in den Vorverhandlungen auf nationaler Ebene. Zusätzlich existiert ein beträchtliches Ungleichgewicht zwischen privaten und öffentlichen Interessen. Der Einfluss spezifischer Produzenteninteressen ist vor allem bei den Vorverhandlungen in Deutschland wichtig, während Finnland dazu tendiert, Konsumenteninteressen zu bevorzugen.

Unsere Modellevaluation basiert auf der Nash-Verhandlungslösung (NVL). Die Spezifikation der einzelnen NVL-Modelle folgt Standarddefinitionen für die unterschiedlichen Interessenvermittlungsausprägungen. Daten zu den Präferenzen der Akteure haben wir dem „National Decision Making in the European Union (NDEU)“ - Datensatz entnommen. Unsere komparative Evaluation der unterschiedlichen Verhandlungsmodelle zeigt, dass die étatistische NVL die höchste Voraussagegenauigkeit hat; im Gegensatz dazu bietet das konkordanzdemokratische Modell die ungenauesten Prognosen. Die Analyse zeigt außerdem wichtige Unterschiede zwischen den vier untersuchten Staaten auf. Eine kompa- 
rative Fallstudie über den Gesetzesvorschlag für „gerichtliche Zuständigkeit und die Anerkennung und Vollstreckung von Entscheidungen in Zivil- und Handelssachen" (Com 99/348) zeigt diese Divergenzen beispielhaft auf.

Dieses Kapitel ist wie folgt aufgebaut: Im nächsten Abschnitt fassen wir die Literatur zur Interessenvermittlung bei der EU-Entscheidungsfindung zusammen, um daraus eine neue Klassifizierung von Interessenvermittlungstypen abzuleiten. Anschließend stellen wir das Forschungsdesign und den Datensatz detaillierter vor. Abschnitt 4 veranschaulicht unsere Methode und Vorgehensweise anhand einer Fallstudie, um im Anschluss die verschiedenen Modellen auf ihre Erklärungskraft hin zu vergleichen. Das Kapitel endet mit einer kritischen $\mathrm{Zu}$ sammenfassung.

\section{Muster der Interessenvermittlung in vier Mitgliedsstaaten}

Die Literatur zur Interessenvermittlung folgt noch immer der Tradition der Pionierarbeiten von Lehmbruch (1967), Schmitter (1974) und Lehmbruch/Schmitter (1982). Die Autoren machen für das kontinentale Westeuropa die These geltend, dass die Beziehung zwischen konkurrierenden Interessengruppen eher korporatistischen als pluralistischen Interaktionsmustern folgt. Obwohl die theoretische Begründung und die empirische Bedeutsamkeit dieser Vermutung noch immer umstritten sind (z.B. Traxler/Kittel 2000), steigt weiterhin die Zahl der Artikel, die die Gründe und Konsequenzen der korporatistischen Vermittlung in Europa und darüber hinaus analysieren. Die zunehmende Bedeutung der EU hat die Diskussion in mannigfacher Weise beeinflusst. Die meisten Studien analysieren die mögliche Transformation von Interessenvermittlung auf EU-Ebene (z.B. Andersen/Eliassen 1993; Greenwood/Aspinwall 1998; Mazey/Richardson 1993; Pedler/van Schendelen 1994). Manche Autoren behaupten, dass die EU zunehmend korporatistische Züge annehme (z.B. Gorges 1996), während andere glauben, dass sich die EU in eine pluralistische Richtung bewege (z.B. Traxler/Schmitter 1995). Crombez (2002) dagegen meint, dass alles in allem keine generalisierbaren Interaktionsmuster erkennbar sein sollten, da die Präferenzprofile innerhalb der verschiedenen Entscheidungsfindungsprozesse $\mathrm{zu}$ sehr variieren. Der Vorgänger dieser agnostischen Haltung ist Freeman (1989). Er warnt, dass Interessenvermittlungsmuster vielleicht für Sektoren beobachtbar seien, aber wahrscheinlich nicht für Staaten oder - wie in unserem Fall - für supranationale Einheiten wie die EU.

Nur eine kleine Zahl von Studien untersucht, wie Interessengruppen versuchen, auf nationaler Ebene auf ihre Regierungen einzuwirken (van Schedelen 1993). Im Licht der wachsenden Wichtigkeit der EU empfindet Kohler-Koch 
(1996) es als ein Paradoxon, dass nationale Interessengruppen bei sensiblen Angelegenheiten aktiv werden. Greenwood/Jordan (1993) glauben, dass in Großbritannien der nationale Einfluss weiterhin dominant sei. Auf der Grundlage einer extensiven Umfrage behauptet Eising (2004: 236) schließlich, dass nationale und EU-Vereinigungen eine deutliche Arbeitsteilung zwischen sich etabliert hätten.

Die große Anzahl konkurrierender Hypothesen erschwert das Urteil darüber, ob die Interessenvermittlung in der EU einem bestimmten Muster folgt. Zusätzlich wird eine solche Evaluation durch den Überfluss an Definitionen der Schlüsselkonzepte erschwert. Am offensichtlichsten ist dieses Problem bei der Kategorie "Korporatismus": Dieser Begriff bezieht sich zumeist auf alle Arten von Interessenskonflikten, die meisten Anwendungen fokussieren jedoch auf die Interaktion zwischen Arbeit und Kapital. In einer gründlichen Evaluation der existierenden Literatur identifiziert Siaroff 22 „Strukturen“, die vermutlich charakteristisch für die korporatistische politische Ökonomie sind, und evaluiert die Signifikanz von 23 konkurrierenden Rankings.

Dem Korporatismuskonzept relativ ähnlich ist das Konkordanzmodell der Interessenvermittlung. Beiden ist gleich, dass mächtige und wichtige Interessengruppen relativ unabhängig von staatlichen Regulierungen ihre politischen Aktivitäten koordinieren; sie unterscheiden sich aber hinsichtlich ihrer Annahme zur Macht des Staates. Die Macht der Regierung ist im korporatistischen System höher als im konkordanzdemokratischen System (Baltz et al. 2005).

Diese Vorüberlegungen sind wesentlich für die vergleichende Modellevaluation, die wir in diesem Aufsatz unternehmen. Unsere Studie erkundet die Voraussagegenauigkeit der verschiedenen Interessenvermittlungsmodelle, die sich für die Analyse der nationalen Vorverhandlungen zur EU-Politik aus der einschlägigen Literatur ableiten lassen. Wenn wir uns als erstes auf die konventionelle Unterscheidung zwischen Korporatismus und Pluralismus beziehen, stimmen die meisten Beobachter $\mathrm{zu}$, dass die vier untersuchten Staaten unterschiedlich klassifiziert werden sollten.

Nach Siaroffs Auswertung von 23 Rankings (Baltz et al. 2005) ist der Korporatismus am ausgeprägtesten in den Niederlanden, gefolgt von Deutschland, Finnland und Großbritannien. Beziehen wir zusätzlich institutionelle Ausprägungen der Staaten mit ein, erscheint die Dichotomie zwischen Mehrheits- und Konsensdemokratien sinnvoll. Lane/Ersson $(1997,1999)^{2}$ werten hierzu verschiedene Rankings aus und stellen fest, dass Finnland unter den vier untersuch-

${ }^{2}$ Die verschiedenen Werte, die Lane/Ersson zitieren, sind folgende: Finnland: 1, 1.65, 1.49, 1.47; Niederlande: $2,1.58,1.69,1.40$; Deutschland: $1 ;-0.11 ;-0.68 ;-0.07$; Großbritannien: 0, -1.3, -1.16, 1.25 . 
ten Staaten die ausgeprägteste Konsensusdemokratie und Großbritannien das klarste Mehrheitssystem sei.

Die Literatur lässt uns erwarten, dass nationale Vorverhandlungen im GroBen und Ganzen den Vermittlungsmustern folgen, die auch in einem Mitgliedstaat vorherrschen können. Das würde bedeuten, dass finnische Interaktionen korporatistisch oder konkordanzdemokratisch sein sollten und dass die Regierung zur Bevorzugung der Arbeiter und Konsumenten tendieren sollte. In den Niederlanden und Deutschland sollte das korporatistische Interessenvermittlungssystem eine Neigung in Richtung der Unternehmens- und Produzenteninteressen zeigen. Zuletzt schlägt die Literatur vor, dass Großbritannien einem pluralistischen Interaktionsmodus folgen sollte.

Im Folgenden werden wir testen, ob diese weitgehend induktiven Generalisierungen der empirischen Realität standhalten. Da Interessenvermittlung zum großen Teil ein Verhandlungsprozess zwischen konkurrierenden Akteuren ist, werden wir verschiedene Verhandlungsmodelle einführen und ihre Voraussagegenauigkeit evaluieren. Weil die Akteurspositionen bei spezifischen Fragen aufeinander bezogen sein sollten, glauben wir, dass eine themenbezogene Analyse einem akteursfokussierten Ansatz vorzuziehen ist. Die Grundlage aller im Folgenden vorgestellten Verhandlungsmodelle ist die Nash-Verhandlungslösung (NVL) (Nash 1950; Bailer/Schneider 2005; Schneider et al. 2006). ${ }^{3}$

\section{Forschungsdesign}

Für die Analyse stützen wir uns auf die Daten aus dem „National Decision Making in the European Union"-Datensatz (NDEU). Diese Quelle bietet detaillierte Informationen über 15 Gesetzesvorschläge, die die Europäische Kommission initiierte und die zwischen 1997 und 1999 den Mitgliedsstaaten zur Abwägung übermittelt wurden. Die NDEU-Fälle beziehen sich auf ein größeres Sample von ca. 70 Gesetzesvorschlägen, die in der Veröffentlichung der „Decision Making in the European Union (DEU)“-Forschungsgruppe komplett analysiert wurden. ${ }^{4}$ Der DEU-Datensatz ist ein vielschichtiges Sample von Gesetzesvorschlägen der Europäischen Kommission von 1997 bis 2000. Gesetzesvorschläge wurden nur dann in beide Datensätze aufgenommen, wenn sie mehreren Kriterien genügten.

\footnotetext{
${ }^{3}$ Bailer/Schneider (2006) und Schneider et al. (2006) verwenden verschiedene Versionen der NVL, um die Voraussagegenauigkeit der konkurrierenden Modelle der EU-Entscheidungsfindung einzuschätzen, insbesondere die Interaktion innerhalb des Ministerrats.

${ }^{4}$ Dieses Großforschungsprojekt evaluierte die Erklärungskraft von konkurrierenden spieltheoretischen Modellen der EU-Entscheidungsfindung. Zusammenfassende Ergebnisse können bei Thomson et al. (2006) sowie Stokman/Thomson (2004) gefunden werden.
} 
Erstens musste das Sample die Vielseitigkeit der Gesetzesprozeduren innerhalb der EU repräsentieren. Zweitens musste der Vorschlag ausreichend kontrovers sein. Drittens mussten die Hauptgebiete der Gesetzgebung abgedeckt sein. Die 15 hier untersuchten Vorschläge beziehen sich auf sehr unterschiedliche Politikfelder: von Gesundheits- über Konsumenten- bis hin zu Fischereipolitik. Tabelle 1 zeigt die unterschiedliche Anzahl strittiger Themen, die diese Vorschläge in den jeweiligen Staaten provoziert haben, die Zahl der Akteure und die Varianz der Policy-Positionen.

Die Daten wurden mittels Experteninterviews und Sekundärquellen gesammelt. Nach einer Vorbefragung über die Kompetenz und Verfügbarkeit der voraussichtlichen Interviewpartner wurden vier Mitarbeiter der Universität Konstanz zu Politikexperten in die Hauptstädte der vier Mitgliedstaaten geschickt, um strukturierte Tiefeninterviews zu führen. Der Forschungsassistent befragte die Experten zu ihren Kenntnissen über die nationalen Verhandlungen, die der Interaktion im Ministerrat vorausgehen. Jeder Experte - einer für jeden Gesetzesvorschlag in jedem Mitgliedsstaat - verfügte über detaillierte Informationen zu den nationalen Vorverhandlungen der jeweiligen Vorschläge. Um verlässliche und valide Informationen zu bekommen, mussten wir zwischen den Informationen, die uns jeder Experte liefern konnte, und der Möglichkeit einer strategisch oder anders motivierten Fehldarstellung abwägen. Deswegen kamen alle interviewten Experten aus der nationalen Verwaltung oder von nichtstaatlichen Organisationen, die an den nationalen Vorverhandlungen teilnahmen, aber nicht eine Führungsposition innerhalb dieser Organisation innehatten. So konnten die Risiken dieser Methode auf ein Minimum reduziert werden.

In einer ersten Interviewphase identifizierten die Experten alle kontroversen Themen eines Vorschlages. Sodann forderte unser Forschungsteam sie auf, die Akteure zu benennen, die im Entscheidungsfindungsprozess aktiv wurden. Des Weiteren mussten sie die Idealpunkte dieser Akteure zu jedem Thema und die endgültige Positionierung des für die Vorverhandlungen zuständigen Ministeriums zu jedem wichtigen Thema lokalisieren. Wenn möglich, sollten sie zusätzlich die Referenzpunkte (die Verhandlungsposition in dem Fall, dass sich die nationalen Akteure nicht auf einen einheitlichen Vorschlag einigen können) und die Bedeutung festlegen, die jeder Akteur einem Vorschlag zuschrieb. 
Tabelle 1: Ein Vergleich der Vorschläge in vier Staaten

\begin{tabular}{|c|c|c|c|c|c|c|c|c|c|c|c|c|c|c|c|}
\hline \multirow[b]{2}{*}{ Vorschlag } & \multicolumn{5}{|c|}{ Anzahl der Themen } & \multicolumn{5}{|c|}{ Zahl der Akteure ${ }^{\prime}$} & \multicolumn{5}{|c|}{ Standardabweichung } \\
\hline & D & Fin & NL & GB & $\varnothing$ & $\mathbf{D}$ & Fin & NL & GB & $\varnothing$ & D & Fin & NL & GB & $\varnothing$ \\
\hline Altwagenverwertung & 2 & 1 & 1 & 5 & 2.3 & 13 & 6 & 6 & 7 & 8 & 37 & 41 & 34 & 47 & 40 \\
\hline $\begin{array}{l}\text { Besteuerung des Sparein- } \\
\text { kommens }\end{array}$ & 3 & 3 & 3 & 5 & 3.5 & 5 & 6 & 5 & 18 & 8.5 & 39 & 44 & 46 & 44 & 43 \\
\hline $\begin{array}{l}\text { Besteuerung von Zigaret- } \\
\text { ten }\end{array}$ & 2 & 1 & 2 & 1 & 1.5 & 5 & 2 & 4 & 5 & 4 & 46 & 71 & 47 & 45 & 52 \\
\hline Beaufsichtigung e-money & 1 & 3 & 3 & 0 & 1.8 & 4 & 5 & 5 & - & 4.7 & 50 & 42 & 48 & - & 47 \\
\hline $\begin{array}{l}\text { Mitteilungsabkommen } \\
\text { Notification agreements }\end{array}$ & 3 & 3 & 5 & 2 & 3.3 & 6 & 8 & 8 & 10 & 8 & 40 & 42 & 43 & 40 & 41 \\
\hline $\begin{array}{l}\text { Juristische Aspekte bei E- } \\
\text { Commerce }\end{array}$ & 3 & 4 & 2 & 2 & 2.8 & 17 & 9 & 7 & 11 & 11 & 30 & 39 & 32 & 43 & 36 \\
\hline $\begin{array}{l}\text { Prävention und Kontrolle } \\
\text { von }\end{array}$ & 1 & 1 & 3 & 0 & 1.3 & 9 & 13 & 6 & - & 9.3 & 38 & 8 & 39 & - & 28 \\
\hline $\begin{array}{l}\text { Prevention/ Control of } \\
\text { TSE }\end{array}$ & & & & & & & & & & & & & & & \\
\hline $\begin{array}{l}\text { Grenzen veterinärmedizi- } \\
\text { nischer Produkte }\end{array}$ & 0 & 0 & 4 & 0 & 1 & - & - & 9 & - & 9 & - & - & 39 & - & 39 \\
\hline Gründung von "Eurodac" & 1 & 0 & 3 & 1 & 1.3 & 3 & - & 6 & 6 & 5 & 53 & - & 49 & 41 & 48 \\
\hline $\begin{array}{l}\text { Nord-Ostatlantische } \\
\text { Fischereizone }\end{array}$ & 0 & 0 & 1 & 0 & 0.3 & - & - & 3 & - & 3 & - & - & 50 & - & 50 \\
\hline $\begin{array}{l}\text { Gerichtl. Zuständigkeit in } \\
\text { Handelssachen }\end{array}$ & 3 & 1 & 3 & 1 & 2 & 11 & 4 & 5 & 7 & 6.8 & 31 & 50 & 43 & 50 & 44 \\
\hline $\begin{array}{l}\text { Dialog gemeinsamer } \\
\text { Fischereien }\end{array}$ & 1 & 2 & 2 & 1 & 1.5 & 10 & 6 & 3 & 3 & 5.5 & 42 & 34 & 49 & 58 & 46 \\
\hline $\begin{array}{l}\text { Schwerfällige Tiere/ } \\
\text { Schwein }\end{array}$ & 0 & 0 & 1 & 1 & 0.5 & - & - & 5 & 5 & 5 & - & - & 46 & 45 & 46 \\
\hline $\begin{array}{l}\text { Gleichbehandlung } \\
\text { Beschäftigung }\end{array}$ & 1 & 2 & 3 & 1 & 1.8 & 11 & 4 & 8 & 7 & 7.5 & 31 & 40 & 35 & 34 & 35 \\
\hline Spielzeug aus PVC & 3 & 3 & 3 & 1 & 2.5 & 10 & 12 & 8 & 8 & 9.5 & 33 & 31 & 41 & 39 & 36 \\
\hline Aggregatswert & 24 & 24 & 39 & 21 & 10 & 22 & 155 & 23 & 169 & 77 & 39 & 40 & 40 & 44 & 41 \\
\hline & & & & & 8 & 0 & & 0 & & 4 & & & & & \\
\hline
\end{tabular}

Bemerkung: Die offiziellen Notationen dieser Gesetzesvorschläge sind, in absteigender Reihenfolge, wie folgt: Com (97) 358; Com (98) 295; Com (98) 320; Com (98) 461; Com (98) 546; Com (98) 586; Com (98) 623; Com (99) 130; Com (99) 260; Com (99) 345; Com (99) 348; Com (99) 382; Com (99) 456; Com (99) 565-7; Com (99) 577

${ }^{1}$ Es wurde die höchste Anzahl von Akteuren bei jedem Thema genommen, in dem Fall, dass die Anzahl der Akteure innerhalb eines Mehr-Themenvorschlags variiert.

Um die Vorschläge vergleichbar zu machen, wurden alle Akteurspositionen und die möglichen Ergebnisse auf einer Skala von 0 bis 100 normiert. Demzufolge ist die diesem Beitrag zugrunde liegende Annahme, dass die bestehenden PolicyKonflikte auf einer kontinuierlichen Skala abgebildet werden können. Ähnlich wie Schneider et al. (2006) beschränkten wir die möglichen Werte der Optimierungsfunktion zum Verhandlungsraum zwischen 0 und 100 und verließen uns 
auf die Constrained Optimization (CO) Routine in GAUSS für die Berechung des Optimums. Wir verwendeten den Newton-Algorithmus als Optimierungsmethode und den Mittelwert als den Anfangsvektor in allen Modellen. ${ }^{5}$ Der Mittelwert wurde durch alle Positionen der nationalen Akteure bei jedem Thema eines Vorschlags berechnet.

Die verschiedenen Interessenvermittlungsmodelle wurden durch die Akteurspositionen wie folgt konstruiert: Das pluralistische Interessenvermittlungsmodell sagt voraus, dass die für die nationalen Vorverhandlungen verantwortlichen Ministerien versuchen, die Interessen aller aktivierten Interessen auszubalancieren und selbst die Mittelposition einzunehmen. Also liegen dem pluralistischen Modell, das wir hier testen, die Positionen aller Akteure zugrunde. Das an die Theorie der Konkordanzdemokratie angelehnte Modell bezieht sich auf die mächtigen Interessengruppen, die ihre Position zu Streitthemen mit dem führenden Ministerium koordinieren. Das Ministerium folgt den Vorstellungen dieser Interessengruppen. Insofern basiert dieses Modell nur auf den Positionen der mächtigsten Akteure links und rechts von der Position des führenden Ministeriums, wohingegen das korporatistische Modell auch die Position dieses entscheidenden Regierungsagenten berücksichtigt.

Das étatistische Modell der Interessenvermittlung bezieht sich nur auf die Position des federführenden Ministeriums und anderer teilnehmender staatlicher Akteure. Im Gegensatz dazu bezieht das klientelistische Modell die Positionen des federführenden Ministeriums und jener Interessengruppen mit ein, die nicht weiter als ein Drittel von der maximal möglichen Distanz auf der linken oder rechten Seite der anfänglichen Position des federführenden Ministeriums entfernt sind. In diesem Modell werden also nur die nahen „Verbündeten“ des staatlichen Akteurs im Agenda-Setting als relevant betrachtet.

Für jedes Interessenvermittlungsmodell schätzen wir eine symmetrische und eine asymmetrische Nash-Verhandlungslösung (NVL). Die symmetrische NVL sagt voraus, dass die Akteure kollektiv eine einzige Lösung im nicht leeren Verhandlungsraum $\Theta$ wählen. Diese Lösung wird durch die Maximierung des Produkts der Nutzenfunktion der Akteure U geschätzt:

$S Y M . N B S=\max _{O \in \Theta} \prod_{i=1}^{n} U_{i}$

mit $\mathrm{i}=1, \ldots, \mathrm{n}$ für die beteiligten Akteure und

$U_{i}=\left|D_{i}\right|-\left|\operatorname{pos}_{i}-o\right|$

\footnotetext{
${ }^{5}$ Um für einen möglichen, aber ungewollten Einfluss des Startvektors auf die Ergebnisse zu kontrollieren, wurden die Modelle mit unterschiedlichen Startvektoren berechnet.
} 
mit $0 \leq$ pos $_{i} \leq 100$ und beschränkt durch die Randbedingung der individuellen Pareto-Effizienz ( $U_{i} \geq$ für alle i).

Hierbei benennt $p^{2} s_{i}$ die Policy-Position von Akteur i und $D_{i}$ ist sein Uneinigkeitswert, während o das Verhandlungsergebnis ist. Deshalb nimmt der Nutzen des Akteurs i zu, je kleiner $\mid$ pos $_{\mathrm{i}}$-ol im Vergleich zu $D_{i}$ ist.

Die asymmetrische NVL fügt die relative Macht der teilnehmenden Akteure im Exponenten hinzu. Wir verwenden hierfür das Machtmaß aus den Experteninterviews.

$$
A S Y M . N B S=\max _{O \in \Theta} \prod_{i=1}^{n} U_{i} p_{i}
$$

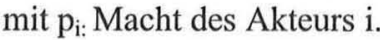

Im Gegensatz zu dem in Bailer/Schneider (2006) präsentierten Modell, aber entsprechend Schneider et al. (2004) setzen wir aus zwei Gründen den Uneinigkeitswert $D_{i}$ nicht mit der Distanz zwischen dem Referenzpunkt (sq) und der Policy-Position des Akteurs (|sq-pos $\mid$ ) gleich. Erstens: Verlässt man sich auf den Referenzpunkt für die Operationalisierung des Uneinigkeitswerts, würde dies - unter der Randbedingung der individuellen Pareto-Effizienz - notwendigerweise für die meisten Fälle implizieren, dass das Ergebnis gleich dem Referenzpunkt ist. Zweitens gilt, dass ein gemeinsamer Referenzpunkt für alle Spieler nicht mit Sicherheit existiert. Aufgrund des komplexen Zwei-Ebenen-Charakters der EU-Politikgestaltung bleibt der Uneinigkeitswert für den individuellen Akteur unsicher.

Um diesen Problemen entgegenzutreten, operationalisieren wir den erwarteten Uneinigkeitswert als die Wahrscheinlichkeit, das Worst-Case-Szenario in dem Fall zu vermeiden, dass keine Einigung erreicht wird. Es wird angenommen, dass diese Wahrscheinlichkeit eine Funktion der relativen Verhandlungsstärke jedes Spielers ist. Der Uneinigkeitswert ist insofern wie folgt definiert:

$$
D_{i}=\left(1-p_{i}\right) * g_{i}
$$

Der Parameter $p_{i}^{6}$ misst die relative Macht des entsprechenden Akteurs I und $g_{i}$ ist die Auszahlung für den schlimmstmöglichen Fall. ${ }^{7}$ Für einige Fälle, bei denen die (individuell pareto-effiziente) Lösungsmenge (das Winset) leer ist, sagt das NVL-Modell generell den Referenzpunkt voraus.

\footnotetext{
${ }^{6}$ Die Summe der Machtanteile ergibt dabei 1: $\sum_{i=1}^{n} p_{i}=1$

${ }^{7}$ Für den Faktor $g_{i}$ gilt dabei die folgende Nebenbedingung: $g_{i}=$ pos $_{i}$ falls pos $\geq 50$ und 100-pos ${ }_{i}$ falls pos $\leq 50$.
} 
Tabelle 2 und Abbildung 1 illustrieren die Berechnung an einem einfachen Beispiel mit drei Akteuren.

Tabelle 2: Die Modellparameter am Beispiel mit drei Spielern

$\begin{array}{llllll}\text { Pos } & \text { p } & \text { g } & \text { D } & \text { o } & \text { U }\end{array}$

\begin{tabular}{|c|c|c|c|c|c|c|}
\hline Spieler 1 & 20 & 0,3333 & 80 & 53,3 & 40 & 33,3 \\
\hline Spieler 2 & 25 & 0,3333 & 75 & 50 & 40 & 35 \\
\hline Spieler 3 & 70 & 0,3333 & 70 & 46,7 & 40 & 16,7 \\
\hline
\end{tabular}

Abbildung 1: Nutzenfunktionen und NVL am Beispiel von drei Spielern

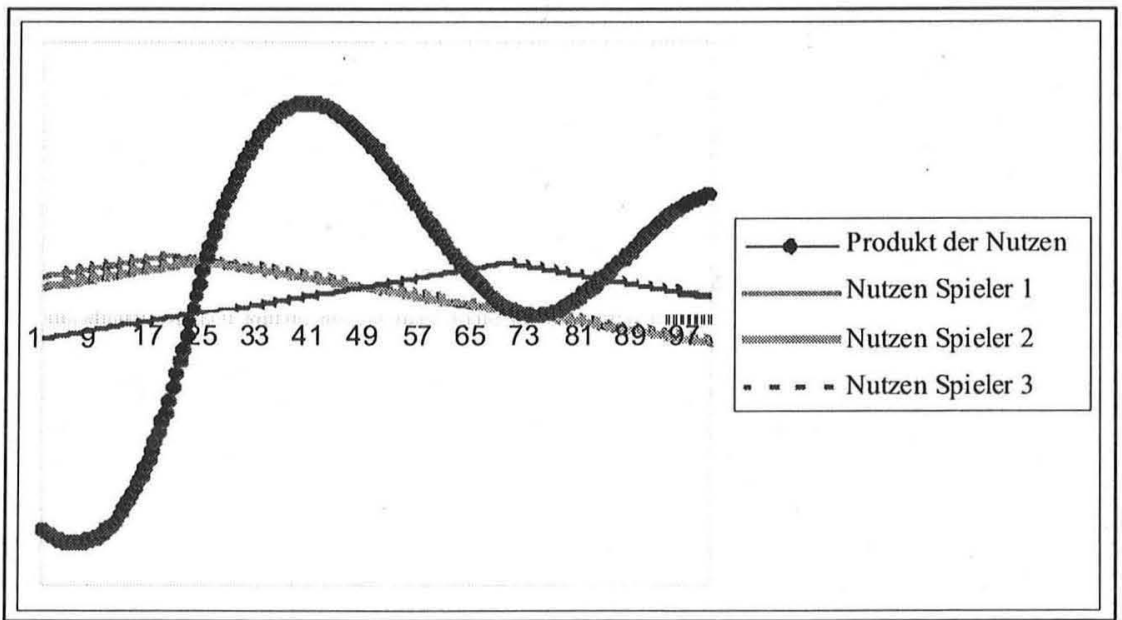




\section{Illustration}

In diesem Kapitel veranschaulichen wir unsere Forschungsstrategie mit einer Fallstudie. Wir haben den Gesetzesvorschlag COM (99) 348 als Beispiel ausgewählt, um unser Forschungsdesign zu veranschaulichen. Der Gesetzesvorschlag betraf die gerichtliche Zuständigkeit und die Anerkennung und Vollstreckung von Entscheidungen in Zivil- und Handelssachen Der Kommissionsvorschlag basierte auf den Konventionen von Brüssel und Lugano, die zum ersten Mal zu Beginn der 1970er Jahre diskutiert wurden. Ein Ziel des Gesetzesprojekts war es, den Spielraum der existierenden, auf diesen Vereinbarungen beruhenden Gesetzgebung auf neue Themen wie elektronischen Handel (E-Commerce) zu erweitern. Der Hauptstreit, den der Kommissionsvorschlag hervorrief, bezog sich auf die verschiedenen Möglichkeiten der gerichtlichen Zuständigkeit. Die Konflikte, die dieser Vorschlag auf nationaler Ebene entstehen ließ, waren jedoch nicht ausschließlich juristischer Natur. Da wichtige Wirtschaftsinteressen auf dem Spiel standen, wurden Produzenten- und Konsumentenorganisationen neben dem führenden Ministerium und anderen staatlichen Akteuren aktiv.

Für jeden Mitgliedstaat werden die Positionen der Akteure zu jeder Streitfrage dargestellt, die diese Gesetzgebungsinitiative hervorrief. Im Falle, dass der Kommissionsvorschlag einen multi-dimensionalen Konfliktraum in einem Mitgliedstaat eröffnete, begrenzen wir unsere Visualisierung der relativen Positionen der Akteure auf das wichtigste Thema des Vorschlags, da wir in diesem Paragraph unsere Vorgehensweise nur beispielhaft illustrieren wollen.

Deutschland: Der Kommissionsvorschlag führte in Deutschland deshalb zu einer besonderen Konstellation, weil sowohl Justiz- als auch Wirtschafts- und Technologieministerium federführend waren. Das ruft analytisch keine Schwierigkeiten hervor, da die Idealpositionen dieser Agendasetzer ohnehin fast identisch waren. Während des "EU-Justizgipfels" in Tampere 1999 favorisierte das Justizministerium eine breitere Gesetzesregulierung durch eine Verordnung. Die Experten identifizierten neun andere Akteure, die während der Vorverhandlungen aktiv wurden: Der Deutsche Anwaltsverein, die Bundesanwaltskammer, die Bundesnotarztkammer, der Bundesverband der Freien Berufe, der DIHT (Deutscher Industrie- und Handelstag), der BDI (Bund Deutscher Industrie), die AGV (Arbeitsgruppe der Verbraucherverbände), die Vereinigung der Medien und die Grüne Partei.

Diese Gruppen stimmten bei drei Themen nicht überein. Der erste Streitpunkt betraf die Frage, welcher Rechtsgrundsatz für den zuständigen Gerichtssitz im Fall von Rechtsstreitigkeiten angewendet werden sollte. Die Produzentenseite (DIHT, BDI) unterstützte das Prinzip des Zielstaates. Im Gegensatz dazu favorisierten die Konsumenten (AGV) und die Grünen das Prinzip des Herkunftslan- 
des. Die Ministerien nahmen eine mittlere, unentschiedene Position ein. Das zweite Thema umfasste das Problem der Haftpflicht. Die Medienvereinigung argumentierte, dass der Internet-Provider verantwortlich sein müsste. Die Konsumentenorganisationen und die Grünen behaupteten, dass die Firmen, die dem Konsumenten einen Service über das Internet anbieten, haften müssten. Das letzte kontroverse Thema bezog sich auf die Reichweite der vorgeschlagenen Regulierung: Die AGV und die Grünen machten sich für eine verbrauchernahe Position stark, der Bundesverband der Freien Berufe und die Medienvereinigung vertraten hingegen eher eine laissez-faire-Haltung.

Nur drei Akteure, die Bundesanwaltskammer, der DIHT und der BDI, setzten sich in offener Lobbyarbeit für ihre Positionen ein. Der Adressat der Kampagnen war immer eines der zwei verantwortlichen Ministerien. Die Experten betonten in den Interviews, dass die Bundesregierung an keinerlei Gesprächen mit irgendeiner nationalen Interessengruppe interessiert war. Die Interessengruppen wurden nur eingeladen, an Anhörungen auf EU-Ebene teilzunehmen.

Abbildung 2 zeigt die relativen Positionen der aktiven Gruppen bei dem wichtigsten Thema in den deutschen Vorverhandlungen und auch eine Vorhersage jedes Modells zur endgültigen Positionierung des federführenden Ministeriums zu diesem Thema (die Möglichkeiten der gerichtlichen Zuständigkeit).

Abbildung 2: Plot der relativen Akteurspositionen und Modellvorhersage für die wichtigsten Themen in Deutschland zu Com 99/348 (Prinzip der Zuständigkeit: Herkunftsstaats- vs. Zielstaatsprinzip oder Nuancen davon)

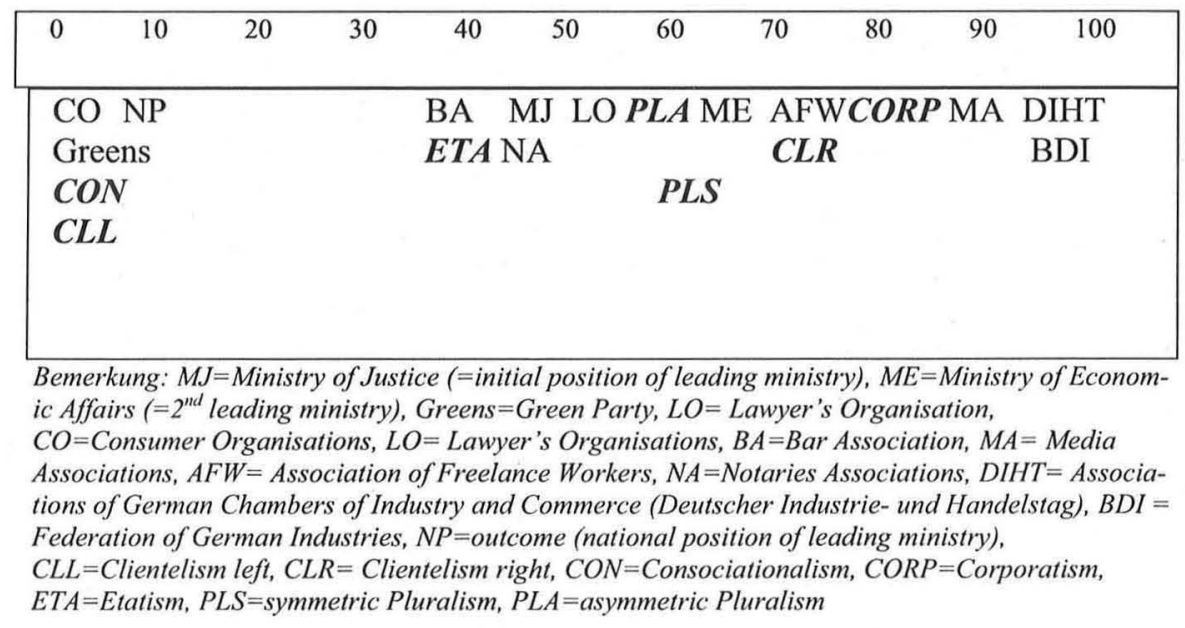


Der Plot zeigt bei diesem Thema in Deutschland, dass das Konkordanzmodell und eines der klientelistischen Modelle dem Ergebnis am nächsten kamen; danach folgt das étatistische Modell. Das Ergebnis messen wir, wie beschrieben, durch die Position des federführenden Ministeriums nach Abschluss der nationalen Vorverhandlungen.

Niederlande: Das Justizministerium war der alleinige Agendasetzer bei diesem Vorschlag. Wie unsere Experten aufzeigten, unterschätzten Unternehmensvereinigungen eher die Signifikanz der Gesetzesvorlage. Sieben verschiedene Gruppen wurden bei mindestens einem der drei untersuchten Themen aktiv.

Die Frage, welches Gericht für Streitigkeiten beim Internethandel verantwortlich sein sollte, verlängerte die Entscheidungsfindung in den Niederlanden. Zwei Koalitionen mit extremen Präferenzen machten es dem federführenden Ministerium ziemlich schwer, eine gemeinsame Position zu finden. Das Wirtschaftsministerium - unterstützt von Industrieorganisationen - kämpfte für das Prinzip des Herkunftsstaates, da diese Allianz steigende Kosten im Falle eines gerichtlichen Konflikts befürchtete, wenn das Zielstaatsprinzip angewendet werden würde. Das andere Lager umfasste das Justizministerium und die Konsumentenorganisationen; diese Koalition zog das Zielstaatsprinzip vor. Das Finanzministerium, das fast indifferent zwischen diesen polarisierten Gruppen stand, beteiligte sich trotzdem an den nationalen Verhandlungen. Das führende Ministerium löste dieses Patt, indem es mit seiner Position näher an die Position des Finanzministeriums rückte.

Andere Themen waren mehr technischer Natur und betrafen zum Beispiel das Problem, ob die Niederlande von siège statutair zu siège réel wechseln sollte oder nicht. Das holländische System in Bezug auf den Sitz eines Unternehmens ist siège statutair (der ständige Sitz des Unternehmens), wohingegen Deutschland, Großbritannien und Belgien dem Prinzip des siège réel folgen, welches den Ort des Unternehmens berücksichtigt, an welchem dieses den Hauptanteil seines Umsatzes realisiert. Nur das holländische Parlament zog einen Wechsel zur anderen juristischen Doktrin vor; alle anderen Akteure (u.a. die Vereinigung der Anwälte, die Staatliche Kommission für Internationales Privatrecht und die Ministerien für Wirtschaftsangelegenheiten und Justiz) lehnten diesen Wechsel ab. Das letzte technische Thema deckte den speziellen Konflikt innerhalb der nationalen Verwaltung zwischen dem Justizministerium und der Staatlichen Kommission für Internationales Privatrecht $\mathrm{ab}$, ob alternativen Prinzipien bzw. Freistellungen oder Ausnahmeregelungen Beachtung geschenkt werden sollte oder nicht. Im Gegensatz zum Justizministerium stellte sich die staatliche Kommission gegen eine Aufnahme von alternativen Prinzipien in den Gesetzentwurf und schaffte es, wenigstens ein paar der vom Ministerium ins Auge gefassten Ausnahmeregelungen oder Freistellungen auszuschließen. 
Das folgende Schaubild verdeutlicht die Positionen aller Akteure und die Vorhersagen, die sich aus den unterschiedlichen Interessenvermittlungsmodellen ableiten lassen.

Abbildung 3: Plot der relativen Akteurspositionen und Modellvorhersage für die wichtigsten Themen in den Niederlanden zu Com 99/348

\begin{tabular}{|lllllr|}
\hline 0 & $20 \quad 30$ & 50 & 80 & 90 & 100 \\
\hline ME & LA NP & CLR & & & CORP Parl \\
MJ & PLS & & & & \\
State Com & PLA & & & & \\
CON & CLL & & & & \\
ETA & & & & & \\
& & & & & \\
& & & & & \\
\end{tabular}

Bemerkung: Manche Akteure waren nicht auf allen drei Streitfragen des Vorschlags aktiv. Es wird angenommen, dass sie zwischen dem nationalen Status Quo und der Position des führenden Ministeriums indifferent sind. Insofern imputierten wir den Mittelwert zwischen dem Referenzpunkt und der Ausgangsposition des führenden Ministeriums als ihre Position bei diesen Themen. Thema 3 hat keinen klaren Referenzpunkt, deswegen wurde die Ausgangsposition des führenden Ministeriums für die Akteure, die bei den anderen Themen aktiv waren aber nicht bei diesem dritten, imputiert. $M J=$ Ministry of Justice (=initial position of leading ministry), ME=Ministry of Economic Affairs, $M F=$ Ministry of Finance, State Com=State Commission for International Private Law, Parl $=$ Parliament, $L A=$ Lawyer's Association, $N P=$ outcome (national position of leading ministry), $C L L=$ Clientelism left, $C L R=$ Clientelism right, $C O N=$ Consociationalism, $C O R P=$ Corporatism, ETA $=$ Etatism, $P$ LS $=$ symmetric Pluralism, $P L A=$ asymmetric Pluralism

Die vergleichende Evaluation der Modellvorhersagen für diesen Vorschlag zeigt eindeutig, dass beide pluralistischen und eines der klientelistischen Modelle dem wirklichen Ergebnis sehr nahe kommen. Es scheint, als unterscheide sich die Verteilung der Akteurspräferenzen zu diesem Thema nicht zwischen den unterschiedlichen Verhandlungsmodellen. Insofern beeinflussten entweder alle oder nur die Akteure, die dem führenden Ministerium am nächsten liegen, das Endergebnis der Entscheidungsfindung. Wie in Deutschland bietet das korporatistische Modell auch in den Niederlanden die schlechteste Vorhersage für diese Streitfrage.

Finnland: Der Kommissionsvorschlag stellt hier lediglich eine Übernahme schon existierender Konventionen in eine EU-Richtlinie dar. Über die Änderungen, die durch diese Transformation entstehen, einigten sich die Mitgliedstaaten bereits 1999. Daher hielt es das Justizministerium nicht für notwendig, eine Anhörung zu organisieren und Interessengruppen zu konsultieren. 
Deswegen waren die einbezogenen Akteure in den Vorverhandlungen nur das Justizministerium, das finnische Parlament, die Handelskammer und der Ombudsmann der Konsumenten. Das einzige Thema, das etwas Konflikt zwischen den verschiedenen Akteuren entstehen ließ, war der Aspekt des Konsumentenschutzes in Bezug auf den länderübergreifenden Internethandel. Hier hätte die ursprünglich nationale gerichtliche Zuständigkeit geändert werden müssen. Das Justizministerium vertrat die Meinung, dass die existierende Formulierung $\left(, 0^{\prime}\right)$ um den Aspekt E-Commerce $(, 100$ ') erweitert werden sollte, da dieser Aspekt ihrer Sicht nach nicht zufrieden stellend abgedeckt war. Das Parlament und der Ombudsmann der Konsumenten stimmten mit dieser Position überein. Die Handelskammer teilte die Sichtweise der anderen Mitgliedsstaaten, d.h. den Referenzpunkt. Das bedeutete, dass aus ihrer Sicht keine Notwendigkeit zu Änderungen in Bezug auf den Aspekt E-Commerce bestand. Da der Kommissionsvorschlag keine substantiell neuen Streitfragen aufwarf, wurden keine Drohungen oder Versprechen während der Verhandlungen ausgesprochen. Abbildung 4 lokalisiert diese Präferenzen sowie die Modellprognosen.

Abbildung 4: Relative Positionen der Stakeholder und Modellvorhersagen für Finnland zu Com 99/348

\begin{tabular}{|lllll|}
\hline 0 & 50,22 & 70,95 & 78,55 & 100 \\
\hline CC & CLL & PLS & PLA & MJ \\
& & & CO \\
& & & P \\
& & & SQ \\
& & & NP \\
& & & CON \\
& & & ETA \\
& & & CLR \\
& & & COR \\
& & & \\
\hline
\end{tabular}

Bemerkung: $C L L=($ Prediction of $)$ Clientelism Left, $C L R=$ Clientelism Right, ETA $=$ Etatism, CON=Consociationalism, COR =Corporatism, PLS = symmetric Pluralism, $P L A=$ asymmetric Pluralism, $N P=$ national position (after pre-negotiations), $S Q=$ Status $Q u o, M J=$ Ministry of Justice (=leading ministry and therefore its initial position), $\mathrm{CO}=$ Consumer Ombudsman, $P=$ Parliament, $C=$ Central Chamber of Commerce

Die Mehrzahl der Verhandlungsmodelle sagt das Ergebnis korrekt voraus oder ist wenigstens nahe dran. Das Modell mit dem höchsten Voraussagefehler ist jenes klientelistische Modell, bei dem die Positionen der wesentlichen Akteure links vom Ministerium liegen. 
Großbritannien: Dieser Fall unterscheidet sich von den anderen drei Mitgliedsstaaten dahingehend, dass das Lord Chancellor's Department (Finanzministerium) der nationale Agendasetzer war und nicht das Justizministerium. Insofern sah die britische Regierung den Gesetzesvorschlag viel mehr als eine finanzielle denn als eine bloße juristische Angelegenheit an. Aber substantielle Streitigkeiten gab es zu diesem Vorschlag in Großbritannien nicht. Ein paar Uneinigkeiten entstanden bei der Frage, ob eine Regulierung überhaupt notwendig sei. Eine andere Diskussion bezog sich auf das auch in den anderen Ländern diskutierte Thema, welches Gericht bei Streitigkeiten über E-Commerce-Verträge zuständig sein sollte. Die allgemeine Regel im internationalen Privatrecht ist, dass das Gericht im Staat des Konsumenten verantwortlich ist. Dieser Tradition steht jedoch das Argument der Anbieter solcher Dienstleistungen entgegen, wonach sich viele Produzenten die Gerichtsprozesskosten in anderen Mitgliedsstaaten nicht leisten könnten. Aber die britische Regierung weigerte sich, um diese Position zu kämpfen, obwohl sie von allen einbezogenen Ministerien darin unterstützt worden wäre. Sie befürchtete, dass eine Diskussion den ganzen Kompromiss, der zwischen den Mitgliedsstaaten schon erreicht wurde, gefährdet hätte. Aber die britischen Akteure einigten sich, jeden Mitgliedsstaat zu unterstützen, der so einen Vorschlag machen würde, wenn sicher gestellt sei, dass dies die einzige Abweichung vom Kompromiss sein würde.

Das einzige übrig gebliebene Thema von Wichtigkeit war, ob Urteile über E-Commerce in die Regulierung eingeschlossen werden sollten oder nicht. ECommerce-Händler, Werbegemeinschaften und die Vereinigung der Britischen Industrie kämpften um den Ausschluss solcher Urteile. Vehement gegen diese liberalistische Position stellten sich die Konsumentenorganisationen und alle aktivierten staatlichen Akteure, eingeschlossen die Handels- und Industrieabteilung, die schottische Verwaltung und das führende Ministerium, das Lord Chancellor's Department. Trotz intensivem Lobbying konnte die E-Commerce-Lobby die Anfangsposition des Lord Chancellor's Department nicht erfolgreich ändern.

Abbildung 5 zeigt die Präferenzen der Akteure und die Lokalisierung der Vorhersagen der Interessenvermittlungsmodelle beim einzigen wichtigen Thema in Großbritannien. Drei Modelle erfassen das Ergebnis richtig: Das étatistische, eines der klientelistischen und das korporatistische Modell, wobei das konkordanzdemokratische vollkommen daneben liegt. 
Abbildung 5: Relative Positionen der Stakeholder und Modellvoraussagen für GB zu Com 99/348

\begin{tabular}{|llllll|}
\hline 0 & 20 & 50 & 58,9 & 61,8 & 100 \\
\hline ET & BI & CLL & PLS & PLA & LC \\
AA & & & & & DTI \\
CON & & & & CO \\
& & & & & SA \\
& & & & & NP \\
& & & & SQ \\
& & & & ETA \\
& & & & COR R \\
& & & \\
\end{tabular}

Bemerkung: $C L L=($ Prediction of) Clientelism Left, CLR=Clientelism Right, ETA=Etatism, $C O N=$ Consociationalism, $C O R=$ Corporatism, $P L S=$ symmetric Pluralism, $P L A=$ asymmetric Pluralism, $N P=$ national position (=outcome of pre-negotiations), $S Q=$ Status Quo, AA=advertising associations, $E T=e$-commerce traders, $B I=$ confederation of British industry, $L C=$ Lord Chancellor's Department (=initial position of leading ministry), DTI=Department of Trade and Industry, $C O=$ consumer organizations, $S A=$ Scottish Administration

Die deskriptiven Ausführungen dieser Fallstudie zeigen, dass sich die Mobilisierung der Akteure im Vergleich der Mitgliedstaaten stark unterscheidet. Die Ergebnisse der Fallstudie lassen überdies erwarten, dass federführende Ministerien mit ihren Vorschlägen weitgehend in den Staaten erfolgreich sind, in denen die Konfliktintensität niedrig war (GB und Finnland). Das étatistische Modell sollte demnach die höchste Vorhersagegüte unter den konkurrierenden Modellen der Interessenvermittlung haben. Andere Erklärungen sollten dagegen erfolgreicher sein, die Ergebnisse in Deutschland und den Niederlanden, wo der Kommissionsvorschlag zu intensiveren Diskussionen führte, vorherzusagen.

Die Resultate aus Tabelle 3 bestätigen diese Erwartungen im Großen und Ganzen. Sie präsentiert die Vorhersage für alle Modelle und ihre Vorhersagefehler bei den strittigen Themen für jedes Interessenvermittlungsmodell und jedes Thema in allen vier Mitgliedsstaaten. Da die Grundlage eine Skala von 0 bis 100 ist, beträgt der maximale Fehler 100. 
Tabelle 3: Voraussage und Voraussagefehler der Interessensvermittlungsmodelle zu COM 99/348

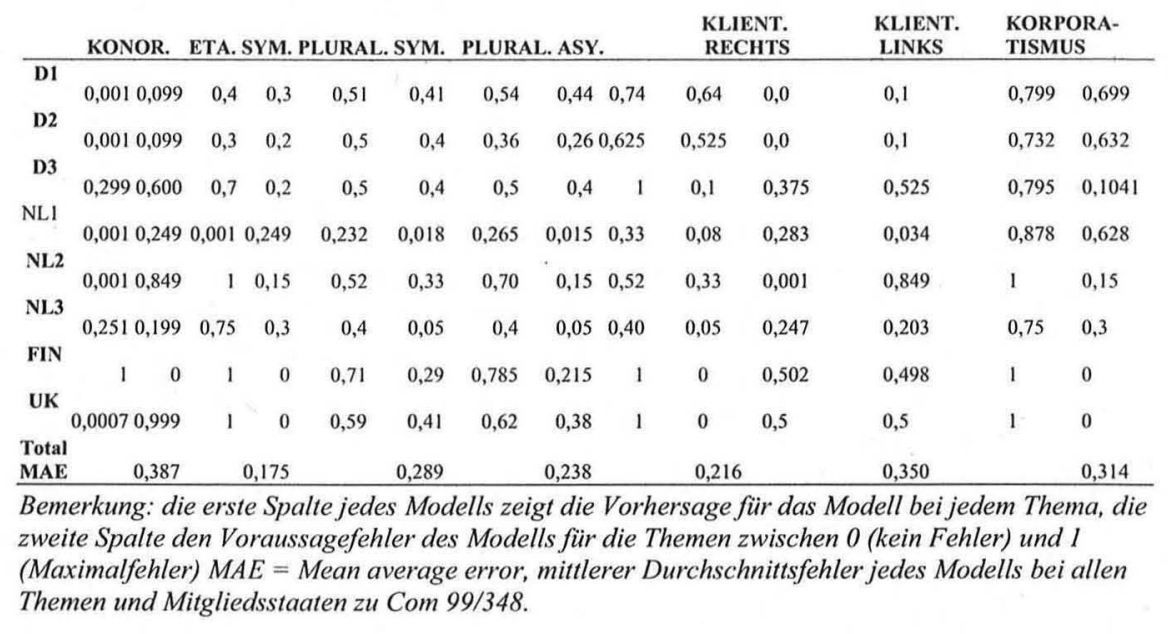

Die Ergebnisse aus Tabelle 3 bestätigen unseren Verdacht, dass das étatistische Modell diesen Vorschlag am besten in Finnland und GB vorhersagt. Die Genauigkeit der korporatistischen und konkordanzdemokratischen Modelle hängt damit zusammen, dass sie fast identisch zum étatistischen Modell sind, da die Anzahl der relevanten nichtstaatlichen Akteure für diesen Fall relativ klein ist. In Deutschland passen jene Modelle, welche die relative Macht der Interessengruppen berücksichtigen, mindestens genauso gut wie das étatistische Modell, in den Niederlanden übertreffen diese asymmetrischen NVL die Vorhersagegüte des étatistischen Modells sogar. Tabelle 3 zeigt auch, dass die verschiedenen Modelle sich in ihrer Vorhersage unterscheiden.

\section{Voraussagegenauigkeit der Interessenvermittlungsmodelle}

In diesem Abschnitt evaluieren wir die Prognosegüte der konkurrierenden Interessenvermittlungsmodelle über alle Themen in allen vier Mitgliedstaaten. In einer früheren Studie haben wir herausgefunden, dass bei den Gesetzesvorschlägen aus unserem Sample kein eindeutiges System der Interessenvermittlung für eines der vier Mitgliedstaaten abgeleitet werden kann (Baltz et al. 2005). Alles in allem scheinen regierungsdominierte Interessenvermittlungssysteme (étatistische Modelle) die Verhandlungen in allen vier Mitgliedsstaaten zu dominieren. Den- 
noch können anderen Arten der Interessenvermittlung, wie etwa die pluralistischen oder korporatistischen Modelle, in fast allen der vier Mitgliedstaaten auf der Ebene einzelner Gesetzesvorschläge gefunden werden. In dieser Studie versuchen wir herauszufinden, ob dieser Eindruck bestätigt werden kann. Wir verwenden verschiedene Evaluationskriterien, um die Voraussagegenauigkeit der Verhandlungsmodelle auf diesen strittigen Themen abzuschätzen. Das erste Maß in unserer Evaluation ist der mittlere absolute Fehler (MAF) der Voraussage zu jedem Thema, welcher ein Standardrichtwert zwischen 0 (kein Fehler) und 100 (maximaler Fehler) ist. Tabelle 4 zeigt die MAFs von sieben verschiedenen Modellen für alle Themen und für alle vier Mitgliedsstaaten separat mit den Maximalfehlern jedes Modells. Zu bemerken ist, dass wir die asymmetrische Version des korporatistischen und konkordanzdemokratischen Modells nicht mit einbeziehen, weil sich die Ergebnisse nicht signifikant von denen der symmetrischen Modelle unterscheiden.

Tabelle 4: Mittlerer Durchschnittsfehler der Modellvoraussage

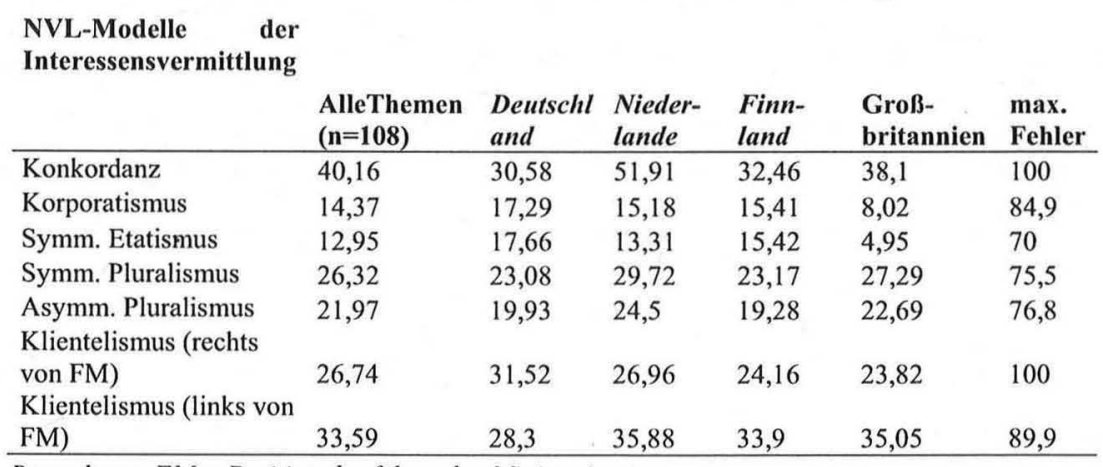

Bemerkung: $F M=$ Position des führenden Ministeriums

Die Ergebnisse aus Tabelle 4 zeigen, dass ein Modell - das symmetrischétatistische Modell - die Daten eindeutig besser abbildet als alle anderen hier untersuchten Modelle. Das korporatistische und das pluralistische Modell, dieMachtungleichheit zwischen den Akteuren berücksichtigen, liefern die nächstbesten Vorhersagen. Das symmetrisch-pluralistische Modell und eines der klientelistischen Modelle liegen dazwischen, während das Konkordanzmodell am Ende der Rangliste steht. Ein Vergleich der beiden klientelistischen Modelle untereinander zeigt, dass vor allem jene Akteure in den nationalen Vorverhandlungen zu gewinnen scheinen, welche zu tiefer gehender Integration neigen als das führende Ministerium. (Ein höherer Wert auf der 100-Punkte-Präferenzskala weist in den meisten Fällen darauf hin, dass ein Akteur eine Intensivierung der 
europäischen Zusammenarbeit befürwortet). Die Rangfolge zwischen den beiden klientelistischen Modellen ist nur in Deutschland umgekehrt. Das bedeutet, dass das deutsche Ministerium, das für die Koordinierung im Vorfeld der EUVerhandlungen zuständig ist, stärker von integrationsskeptischen Gruppen beeinflusst wird als die staatlichen Akteure in anderen Staaten. Dies deutet auf einen bestimmten politischen Bias hin: Da "Integration" oft eher für konsumenten- als für produzentenfreundliche Gesetzgebung steht, scheint die deutsche Regierung oftmals eher dem Druck der Industrielobby nachzugeben als dies andernorts der Fall ist (Schneider/Baltz 2003a, b).

Die Evaluierung unterstreicht außerdem die Bedeutung der staatlichen Akteure in der EU-Politik. Der Unterschied in der Voraussagegenauigkeit des konkordanzdemokratischen und des korporatistischen Modells unterstreicht dies. Die beiden Modelle unterscheiden sich nur insofern, als dass das erste das federführende Ministerium ausschließt und das zweite es als relevanten Akteur mit einschließt. Eine detaillierte Analyse der Agenda-Setting-Kapazität der staatlichen Akteure bestätigt, dass das formal zuständige Ministerium eine beträchtliche Ermessensfreiheit besitzt (Schneider/Baltz 2005).

Bemerkenswert ist auch, dass die relativen Unterschiede zwischen den Modellen über alle vier untersuchten Mitgliedsstaaten hinweg generalisierbar sind. Sogar in den Staaten, von denen ausgegangen wird, dass sie ein korporatistisches Interessenvermittlungssystem haben, bietet das étatistische Modell im Durchschnitt die genaueste Vorhersage. Da das federführende Ministerium in Großbritannien oft seine Ausgangsposition nicht ändern musste, sind das korporatistische und besonders das étatistische Modell hier besonders erfolgreich.

Dies bedeutet, dass in Großbritannien staatliche Akteure den nationalen Verhandlungsprozess dominieren. Es hat auch nicht den Anschein, als sei Großbritannien pluralistischer als Deutschland oder die Niederlande, was der Lehrbuchklassifizierung der in diesen Staaten vorherrschenden Interessenvermittlungssysteme entgegensteht. ${ }^{8}$

Wie Bueno de Mesquita (2004) kürzlich argumentierte, weist der MAF einige Mängel als Maßstab für Modellevaluationen auf. Beispielsweise wird der Prognosefehler eines Modells insofern unterschätzt, als dass der MAF nicht für die unterschiedliche Größe des maximalen Fehlers korrigiert. Der MAF berücksichtigt auch keine Grenz- oder Plafond-Effekte.

\footnotetext{
${ }^{8}$ Hinsichtlich des Referenzpunktes unterscheiden sich die Ergebnisse nur leicht: Die beste Voraussage bietet immer noch das étatistische Modell (MAF bei allen Themen $=0.16$ ), knapp gefolgt vom klientelistischen Modell, das nur die Akteure rechts von der Position des führenden Ministeriums mit einbezieht $(0.165)$ und das asymmetrisch pluralistische Modell $(0.21)$. Den Gewinnern dieses Wettbewerbs folgen das klientelistische Modell mit Gruppen links von dem führenden Ministerium (0.23) und das symmetrisch pluralistische Modell (0.27). Das konkordanzdemokratische Modell liefert immer noch die schlechteste Voraussage (0.29).
} 
Insofern können wir erwarten, dass MAF sowohl negativ als auch positiv mit der Zahl der alternativen Positionen bei einem Thema korreliert. Wenn zum Beispiel nur die extremen Positionen auf einem Themenkontinuum besetzt sind ( 0 und 100) und die Voraussage bei 50 liegt, dann kann der maximale Fehler nur bei 50 Punkten liegen. Werden mehr als zwei (extreme) Positionen benannt (z.B. drei bei 0,75 und 100) und die Voraussage ist 75, dann kann der maximale Fehler nur 25 sein (wenn das Ergebnis 100 ist) oder 75 (wenn das Ergebnis am anderen Extrem der Präferenzskala liegt).

Ein zweites Richtmaß - die Anzahl der Punktprognosen - vermeidet diese Probleme. Tabelle 5 zeigt die Voraussagegenauigkeit der Modelle anhand dieses Maßes. Wir unterscheiden drei „Fehlergrenzen“. Das erste Toleranzniveau erlaubt Abweichungen von $0.1 \%$ der gesamten Verhandlungszone, das zweite weitet dies auf $1 \%$ aus und das dritte Kriterium auf $10 \%$.

Tabelle 5: Anzahl der Punktvorhersagen

\begin{tabular}{|c|c|c|c|}
\hline \multirow[b]{2}{*}{ NVL-Modell } & \multicolumn{3}{|c|}{ Abweichung (Fehlerniveau) } \\
\hline & $\leq 0.1 \%$ & $\leq 1 \%$ & $\leq 10 \%$ \\
\hline Konkordanz & $24(22.22 \%)$ & $26(24.07 \%)$ & $37(36.11 \%)$ \\
\hline Korporatismus & $47(43.51 \%)$ & $47(43.51 \%)$ & $63(58.33 \%)$ \\
\hline Symmetrischer Etatismus & $49(45.37 \%)$ & $49(45.37 \%)$ & $67(62.04 \%)$ \\
\hline Symmetrischer Pluralismus & $2(1.85 \%)$ & $4(3.7 \%)$ & $30(27.78 \%)$ \\
\hline Asymmetrischer Pluralismus & $13(12.04 \%)$ & $15(13.89 \%)$ & $45(41.67 \%)$ \\
\hline Klientelismus (links von FM) & $19(17.59 \%)$ & $19(17.59 \%)$ & $39(36.11 \%)$ \\
\hline Klientelismus (links von FM) & $4(3.7 \%)$ & $4(3.7 \%)$ & $25(23.15 \%)$ \\
\hline
\end{tabular}

Die Ergebnisse aus Tabelle 5 unterscheiden sich leicht im Vergleich zu den MAF-Evaluationen. Obwohl das symmetrisch-étatistische Modell weiterhin die genaueste Vorhersage bietet, ändert sich die Rangfolge etwas. Das klientelistische Modell mit Gruppen links von der Position des federführenden Ministeriums und das symmetrisch-pluralistische Modell leisten schlechtere Voraussagen - das symmetrisch-pluralistische Modell ist nun am Ende der Rangfolge. Sie weisen viel weniger ,perfekte Treffer" - Punktvorhersagen innerhalb der restriktiven $0.1 \%$-Fehlergrenze - als alle anderen Modelle auf; dieses Ergebnis ist unabhängig von der Wahl des Fehlerniveaus. Es zeigt, dass die Regierungsagenten eher nachgeben, wenn rechts und nicht links von ihnen ein wichtiger Akteur ist. Das korporatistische Modell erzielt das zweitbeste Ergebnis von allen Modellen. Das Ergebnis des klientelistischen Modells mit Gruppen rechts von der Posi- 
tion des führenden Ministeriums schneidet fast genauso gut ab. Diese Variante des klientelistischen Modells wird von dem asymmetrisch pluralistischen Modell nur beim $10 \%$-Fehlerniveau übertroffen. Tabelle 5 lässt wieder vermuten, dass die Vorhersagen des konkordanzdemokratischen Modells relativ ungenau bleiben; Regierungsakteure sind, anders gesagt, für unser Verständnis der EUInteressenvermittlung auf nationaler Ebene notwendig. Die Fehler der pluralistischen und klientelistischen Modelle sind mehr um ein „Durchschnittsfehlerniveau" herum konzentriert. Diese Verhandlungsmodelle bieten eine geringere Anzahl von ,perfekten Treffern“ als das korporatistische Modell, aber sie haben auch einen niedrigeren Maximalfehler als die konkordanzdemokratischen Modelle. ${ }^{9}$

Diese Ergebnisse sollten jenen Wissenschaftlern zu denken geben, die Schlussfolgerungen über Interessenvermittlungssysteme eines Staates anhand von wenigen Einzelfallsstudien ziehen. Oft basieren diese auf nur einem Politikfeld oder einer Gesetzesvorlage. Wie unsere Analyse aufzeigt, missachten solche Generalisierungen, dass die europäische Gesetzgebung ein breites Feld an Akteuren über Staaten und Gesetzesvorschlägen hinweg betrifft. Interessenvermittlung kann also in ein und demselben Staat einmal pluralistisch, ein anderes Mal klientelistisch sein, oder die Verhandlungen können ausschließlich unter staatlichen Akteuren stattfinden. Unsere empirischen Befunde deuten des Weiteren darauf hin, dass ein étatistisches Verhandlungsmodell das wirkliche Ergebnis im Durchschnitt am genauesten vorhersagt. Konkordanzmuster sind hingegen seltener als uns manche Interpretationen erwarten lassen.

Uns interessiert nicht nur, welches der unterschiedlichen Modelle die besten Voraussagen des Verhandlungsresultats liefert. Unser Ziel ist es auch, die Variationen in der Voraussagegenauigkeit der Modelle zu verstehen. Daher schätzen wir multivariate lineare Regressionsmodelle, um die Erfolgsbedingungen zu identifizieren, unter welchen manche Modelle besser vorhersagen als andere. Unsere abhängige Variable ist die Distanz zwischen der Voraussage eines Models und dem Verhandlungsergebnis auf Ebene der einzelnen Gesetzesvorschläge, d.h. der Voraussagefehler jedes Modells bei einem Thema. Wie Schneider et al. (2006) untersuchen wir ferner, ob Eigenschaften des Themas und/oder des Vorschlags den Modellvorhersagefehler determinieren.

Wir analysieren auf der Vorschlagsebene, ob die Anzahl der strittigen Themen den Vorhersagefehler der Modelle bestimmt. Die Erwartung ist, dass je mehr Themendimensionen ein Vorschlag hat, desto schwieriger könnte es für die ein-

${ }^{9}$ Ein drittes und für den direkten Vergleich zwischen den Modellen nützliches Maß ist die Anzahl, wie oft ein Modell das Ergebnis besser voraussagt als ein anderes. Wir präsentieren die Ergebnisse von diesem Voraussagefehlermaß nicht, weil die Ergebnisse im Allgemeinen die des MAF-Maßes bestätigen. 
dimensionalen Modelle sein, die Ergebnisse vorherzusagen. In einem multidimensionalen Themenraum ist ein Stimmenhandel möglich, wenn sich die Wichtigkeit der einzelnen Themen unterscheidet. Die nächste Gruppe von erklärenden Variablen verwendet Informationen auf Ebene der einzelnen Streitfragen. Eine erste Variable für die Leistung des Modells, die leicht zu interpretieren ist, stellt die Varianz der Präferenzen bei einem Thema dar. Intuitiv ist zu erwarten, dass eine höhere Variation der Akteurspräferenzen die allgemeine Voraussagegenauigkeit der Modelle verringert. Wir operationalisieren diese Variable durch die Standardabweichung der Idealpositionen. Sie berücksichtigt implizit auch die Zahl der Akteure, weil wir eine Korrelation zwischen der Varianz der Präferenzen bei einem Thema und der Zahl der aktiven Akteure bei dem gleichen Thema erwarten.

Wir erwarten außerdem, dass Ergebnisse leichter vorherzusagen sind, wenn die Präferenzverteilung schief ist. Das Verhandlungsergebnis sollte in diesem Fall näher an der größeren Koalition sein als an der kleineren. Eine weitere Variable misst, ob die Präferenzen zwischen den Akteuren polarisiert sind. Eine Verteilung der Präferenzen kann gleichzeitig etwas schief und stark polarisiert sein, wenn zum Beispiel nur zwei Akteure eine Position an den entgegengesetzten extremen Seiten auf der Präferenzskala aufweisen. Wir messen die Polarisierung der Präferenzen durch den umgekehrten HirschmannKonzentrationsindex. Der Polarisierungsindex erhält den maximalen Wert, wenn die Konzentration niedrig ist. Dies bedeutet, dass alle Quartile (oder nur die besetzten) auf der Themenskala von dem gleichen Anteil an Akteuren besetzt ist. Der Minimalwert steht für den Fall, dass alle Akteure im gleichen Quartil angesiedelt sind; die Präferenzverteilung ist in diesem Fall stark konzentriert. Zuletzt kontrollieren wir für die gegebenen Unterschiede zwischen den untersuchten Mitgliedstaaten, welche die Unterschiede im Voraussagefehler zwischen den verschiedenen Modellen erklären könnten. Laut konventioneller Erkenntnis sollten wir erwarten, dass das pluralistische Modell einen systematisch niedrigeren Voraussagefehler im Fall Großbritanniens als in den anderen drei Mitgliedstaaten haben sollte, und umgekehrt sollten die anderen Interessenvermittlungsmodelle einen höheren Voraussagefehler in Großbritannien im Gegensatz zu Deutschland, Finnland und der Niederlande haben.

Die in Tabelle 5 dargestellten Ergebnisse deuten bereits darauf hin, dass die vorher beschriebenen substantiellen Ergebnisse keinem dieser Verzerrungseffekte zuzuschreiben sind. Das lineare Regressionsmodell schließt eine DummyVariable mit ein, die Großbritannien als ein Beispiel für Pluralismus gegenüber den anderen drei Staaten, die als korporatistischer angenommen werden, darstellt. Sie hat den Wert 0, ist der Mitgliedstaat Großbritannien, anderenfalls 1. 
Tabelle 6 zeigt die Ergebnisse der Regressionsanalyse für alle analysierten Interessenvermittlungsmodelle.

Tabelle 6: Determinanten der Vorhersagefehler von NVL-Modellen der Interessensvermittlung

\begin{tabular}{|c|c|c|c|c|c|c|c|}
\hline & Konkor. & Etatismus & $\begin{array}{l}\text { Plura- } \\
\text { lismus }\end{array}$ & $\begin{array}{l}\text { Asym. } \\
\text { Plur. }\end{array}$ & $\begin{array}{l}\text { Links } \\
\text { Klient. }\end{array}$ & $\begin{array}{l}\text { Rechts } \\
\text { Klient. }\end{array}$ & $\begin{array}{l}\text { Korpora- } \\
\text { tismus }\end{array}$ \\
\hline \multicolumn{8}{|l|}{ Vorschlagsebene } \\
\hline$\overline{\text { Anzahl der Themen }}$ & $\begin{array}{l}0.01 \\
(0.04)\end{array}$ & $\begin{array}{l}-0.01 \\
(0.01)\end{array}$ & $\begin{array}{l}-0.02 \\
(0.03)\end{array}$ & $\begin{array}{l}-0.01 \\
(0.03)\end{array}$ & $\begin{array}{l}-0.05 * * \\
(0.02)\end{array}$ & $\begin{array}{l}0.02 \\
(0.03)\end{array}$ & $\begin{array}{l}-0.01 \\
(0.02)\end{array}$ \\
\hline \multicolumn{8}{|l|}{ Themenebene } \\
\hline $\begin{array}{l}\text { Varianz der Präfe- } \\
\text { renzen }\end{array}$ & $\begin{array}{l}\overline{0.01^{* * *}} \\
(0.00)\end{array}$ & $\begin{array}{l}-0.00 \\
(0.00)\end{array}$ & $\begin{array}{l}0.00 \\
(0.00)\end{array}$ & $\begin{array}{l}0.00 \\
(0.00)\end{array}$ & $\begin{array}{l}0.00 \\
(0.00)\end{array}$ & $\begin{array}{l}0.00 \\
(0.00)\end{array}$ & $\begin{array}{l}-0.00 \\
(0.00)\end{array}$ \\
\hline $\begin{array}{l}\text { Schiefe der Präfe- } \\
\text { renzverteilung }\end{array}$ & $\begin{array}{l}-0.03 * * \\
(0.02)\end{array}$ & $\begin{array}{l}-0.01 \\
(0.01)\end{array}$ & $\begin{array}{l}0.00 \\
(0.01)\end{array}$ & $\begin{array}{l}-0.00 \\
(0.01)\end{array}$ & $\begin{array}{l}0.00 \\
(0.01)\end{array}$ & $\begin{array}{l}-0.00 \\
(0.01)\end{array}$ & $\begin{array}{l}-0.00 \\
(0.01)\end{array}$ \\
\hline Polarisierung & $\begin{array}{l}0.36^{*} \\
(0.20)\end{array}$ & $\begin{array}{l}0.21^{*} \\
(0.12)\end{array}$ & $\begin{array}{l}0.17 \\
(0.14)\end{array}$ & $\begin{array}{l}0.27 * * \\
(0.12)\end{array}$ & $\begin{array}{l}0.39 * * * \\
(0.12)\end{array}$ & $\begin{array}{l}0.37 * * * \\
(0.13)\end{array}$ & $\begin{array}{l}0.21^{* *} \\
(0.10)\end{array}$ \\
\hline \multicolumn{8}{|l|}{ Staatsebene } \\
\hline GB vs. Rest & $\begin{array}{l}-0.03 \\
(0.10)\end{array}$ & $\begin{array}{l}0.06^{* *} \\
(0.03)\end{array}$ & $\begin{array}{l}-0.02 \\
(0.07)\end{array}$ & $\begin{array}{l}-0.04 \\
(0.07)\end{array}$ & $\begin{array}{l}-0.03 \\
(0.06)\end{array}$ & $\begin{array}{l}-0.04 \\
(0.06)\end{array}$ & $\begin{array}{l}0.02 \\
(0.07)\end{array}$ \\
\hline Konstante & $\begin{array}{l}-0.20 \\
(0.18)\end{array}$ & $\begin{array}{l}0.07 \\
(0.09)\end{array}$ & $\begin{array}{l}0.17 \\
(0.17)\end{array}$ & $\begin{array}{l}0.13 \\
(0.16)\end{array}$ & $\begin{array}{l}0.19^{* *} \\
(0.09)\end{array}$ & $\begin{array}{l}-0.02 \\
(0.13)\end{array}$ & $\begin{array}{l}0.09 \\
(0.11)\end{array}$ \\
\hline $\mathrm{N}$ & 108 & 108 & 108 & 108 & 108 & 108 & 108 \\
\hline Adj. $R^{2}$ & 0.12 & 0.08 & 0.00 & $\overline{0.00}$ & 0.05 & 0.07 & $\overline{0.01}$ \\
\hline
\end{tabular}

Bemerkung: Standardfehler in Klammern, ${ }^{*}=$ signifikant auf $1 \%$-Niveau, $* *=$ signifikant auf $5 \%$ Niveau, $* * *=$ signifikant auf $10 \%$-Niveau

Wie Tabelle 6 entnommen werden kann, ist die erklärte Varianz aller Modelle stets relativ klein. Trotzdem zeigt die Analyse ziemlich klar, dass die Polarisierungsvariable einen starken und signifikanten Einfluss über fast alle hier diskutierten Modelle hinweg ausübt. Je polarisierter die Präferenzverteilung ist, desto ungenauer sind die Modellvorhersagen. Die Varianz- und Schiefemaße sind nur im Hinblick auf das konkordanzdemokratische Modell signifikant. Die Staatsebenenvariable dagegen hat nur im étatistischen Modell eine signifikante Auswirkung. Wie schon angedeutet, ist die Agenda-Setting-Kapazität des führenden Ministeriums in Großbritannien beträchtlich. Staatliche Akteure profitieren davon, dass sich alle Gruppen gemeinsam bekämpfen, was sie als folgenschwere Kommissionsvorschläge wahrnehmen. Bei den anderen Modellen ist der Voraussagefehler für Großbritannien höher als für die anderen Staaten, jedoch 
nicht signifikant. Die einzige Variable, die in direktem Gegensatz zu der erwarteten Beziehung zu stehen scheint, ist die Variable „Themenanzahl“. Das klientelistische Modell, das die Gruppen links von der Position des führenden Ministeriums mit einschließt, wird von diesem Faktor beeinflusst. Das widerspricht unserer Erwartung, dass der Handel mit Stimmen zwischen den Akteuren mit zunehmender Zahl an Dimensionen wahrscheinlicher wird. Da aber der Datensatz nur wenige Vorschläge einschließt, bei denen die gleichen Akteure auf mehreren Themen eines einzelnen Vorschlags aktiv werden, ist die Möglichkeit, dass Verhandlungspakete über verschiedene Themen hinweg innerhalb eines Vorschlags oder zwischen verschiedenen Vorschlägen abgeschlossen werden können, relativ klein.

\section{Schlussfolgerung}

In diesem Kapitel untersuchten wir, inwiefern die konventionelle Klassifizierung der Interessenvermittlungssysteme von vier Mitgliedstaaten der EU sinnvoll ist, um die nationale Verhandlungsposition des verhandelnden Ministeriums auf Ratsebene vorherzusagen. Erstens zeigt unsere empirische Analyse, dass die Muster der Interessenvermittlung bei den nationalen Vorverhandlungen weniger scharf getrennt sind, als von der Literatur zu Interessenvermittlungssystemen angenommen. Die Aktivierungs- und Koalitionsmuster zwischen den staatlichen und nichtstaatlichen Akteuren unterscheiden sich zwischen den verschiedenen Vorschlägen so sehr, dass allgemeine Typologien nur bis zu einem gewissen Wert die nationalen Vorverhandlungen erklären können. Das unterstützt die implizite Unmöglichkeitsthese von Crombez (2002) und Freeman (1989), wonach fast keine Generalisierungen über Interessenvermittlung möglich sind. Zweitens unterstreicht unsere Analyse, dass staatliche Akteure die nationalen Verhandlungen bei den meisten Themen dominieren. Sind aber nichtstaatliche Akteure mit einbezogen, ist ein korporatistisches Verständnis der Verhandlungen viel hilfreicher als ein konkordanzdemokratisches. Drittens hängt der Voraussageerfolg des korporatistischen Modells stark von der Einbeziehung staatlicher Akteure ab. Das ist ein herber Schlag für die Befürworter der These, nach welcher das Interessenvermittlungssystem auf eine Ära zuschreitet, in der starke Verbündete ein Gegengewicht zum Einfluss der nationalen Regierungen bilden können. Obwohl Regierungen die Interessen ihrer Prinzipale zu einem gewissen Grad respektieren müssen, besitzen sie beträchtliche und weitgehend unkontrollierte Ermessensfreiheit bei der Formierung ihrer Position zu EUGesetzesinitiativen. 


\section{Literatur}

Andersen, Svein S. /Eliassen, Kjell A. (1993): Making Policy in Europe. The Europeification of National Policy-Making. London: Sage

Bailer Stefanie/Schneider, Gerald (2006): Schelling versus Putnam? The Importance of Constraints in European Legislation. In: Thomson, Robert/Stokman, Frans N./Achen, Cristopher/König, Thomas (Hrsg.) The European Union Decides. Cambridge: Cambridge University Press. 153177

Baltz, Konstantin./König, Thomas/Schneider, Gerald (2005): Immer noch ein etatistischer Kontinent: Die Formierung von Positionen zu EU-Verhandlungen in vier Mitgliedsstaaten. In: Eising, Rainer/Kohler-Koch, Beate (Hrsg.) Interessenpolitik in Europa. Baden-Baden: Nomos 283-309

Bogaards, Matthijs /Crepaz, Markus M.L. (2002): Forum Section: Consociational Interpretations of the European Union. In: European Union Politics 3 2002. 357-381

Bueno de Mesquita, Bruce (2004): Decision Making Models, Rigor and New Puzzles. In: European Union Politics 5 2004. 125-138

Crombez, Christophe (2002): Information, Lobbying and the Legislative Process in the European Union. In: European Union Politics 3 2002. 7-32

Eising, Rainer (2004): Multilevel Governance and Business Interests in the European Union. In: Governance 17 2004. 211-245

Freeman, John R. (1989): Democracy and markets: the politics of mixed economies. Ithaca: Cornell University Press

Gillingham, John (1991): Coal, Steel, and the Rebirth of Europe, 1945-1955: The Germans and French from Ruhr Conflict to Economic Community. Cambridge: Cambridge University Press

Gillingham, John (2003): European Integration, 1950-2003: Superstate or new market economy? Cambridge: Cambridge University Press

Gorges, Michael J. (1996): Euro-Corporatism? Interest Intermediation in the European Community. Lanham: University Press of America

Greenwood, Justin/Aspinwall, Marc D. (Hrsg.) (1998): Collective Action in the European Union: Interests and the New Politics of Associability. London: Routledge

Greenwood, Justin/Jordan, Grant (1993): The United Kingdom: A Changing Kaleidoscope. In: van Schendelen, Marinus P. C. M. (Hrsg.) (1993): National Public and Private Lobbying. Aldershot: Dartmouth. 65-90

Grote, Jürgen R./Schmitter, Philippe C. (1999): The Renaissance of National Corporatism: Unintended Side-effect of European and Monetary Union or calculated Response to the Absence of European Social Policy? In: Transfer 1 1999. 34-63

Haas, Ernst B. (1958): The Uniting of Europe: Political, Social, and Economic Forces 1950-1957. Stanford: Stanford University Press

Haas, Ernst B. (1964): Beyond the Nation-State: Functionalism and International Organization. Stanford: Stanford University Press

Kohler-Koch, Beate (1996): Die Gestaltungsmacht organisierter Interessen In: Jachtenfuchs, Markus/Kohler-Koch, Beate (Hrsg.) (1996): Europäische Integration. Opladen: Westdeutscher Verlag. 193-222

Lane, Jan-Erik/Ersson, Svante (1997): The Institutions of Konkordanz and Corporatism: How Closely are they Connected? In: Swiss Political Science Review 3 1997. 5-29

Lane, Jan-Erik/Ersson, Svante (1999): Politics and Society in Western Europe. London: Sage

Lehmbruch, Gerhard (1967): Proporzdemokratie: Politisches System und politische Kultur in der Schweiz und in Österreich. Tübingen: Mohr.

Lehmbruch, Gerhard/Schmitter, Philippe C. (1982): Patterns of corporatist policy-making. London

Mazey, Sonia/Richardson, Jeremy (Hrsg.) (1993): Lobbying in the European Community. Oxford

Nash, John F. (1950): The Bargaining Problem. In: Econometrica 18. 155-62 
Pedler, Robin/van Schendelen, Marinus P. C. M. (1994): Lobbying the European Union: companies trade associations and interest groups. Aldershot: Dartmouth

Sandholtz, Wayne/Zysman, John (1989): 1992: Recasting the European Bargain. In: World Politics 42 1989. 95-128

van Schendelen, Marinus P. C. M. (Hrsg.) (1993): National Public and Private EC Lobbying. Aldershot: Dartmouth

Schmitter, Philippe C. (1974): Still the Century of Corporatism? In: Review of Politics 36 1974. 85 121

Schneider, Gerald/Baltz, Konstantin (2003a): The Power of Specialization: How Interest Groups Influence EU-Legislation. In: Rivista di Politica Economica 93 2003. 253-83

Schneider, Gerald/Baltz, Konstantin (2003b): Am Gängelband der Verbände: Zum Einfluss von Partikularinteressen auf die deutsche EU-Politik. In: Zeitschrift für Europa- und Staatswissenschaften 1 2003. 199-219

Schneider, Gerald/Finke, Daniel/Bailer, Stefanie (2006): Bargaining Power in the European Union An Evaluation of Competing Game-Theoretic Models. Mimeo, Universität Konstanz

Schneider, Gerald/Baltz, Konstantin (2005): Domesticated Eurocrats: Bureaucratic Discretion in the Legislative Pre-Negotiations of the European Union. In: Acta Politica 40 2005. 1-27

Schneider, Gerald/Finke, Daniel/Baltz, Konstantin (2007): With A Little Help from the State. Interest Intermediation in the Domestic Pre-Negotiations of EU Legislation In: Journal of European Public Policy 14 2007. 444-459

Stokman, Frans/Thomson, Robert (2004): Winners and Losers in the European Union. In: European Union Politics 5 2004. 5-23

Thomson, Robert/Stokman, Frans N./Achen, Cristopher/König, Thomas (2006): The European Union Decides. Cambridge: Cambridge University Press

Stokman, Frans N./Thomson, Robert (Hrsg.) (2004): The Winners and Losers of European Integration. In: Special Issue of European Union Politics 52004

Traxler, Franz/Kittel, Bernhard. (2000): The Bargaining System and Performance: A Comparison of 18 OECD Countries. In: Comparative Political Studies 33 2000. 1154-90

Traxler, Franz/Schmitter, Philippe C. (1995): The Emerging Euro-Polity and Organized Interest. In European Journal of International Relations 1 1995. 191-218 